\title{
ON A METHOD TO RESOLVE THE NUCLEAR ACTIVITY IN GALAXIES AS APPLIED TO THE SEYFERT 2 GALAXY NGC 1358*
}

\author{
Per Olof Lindblad ${ }^{1}$, Kambiz Fathi ${ }^{1,2}$, Maja Huelm ${ }^{1}$, and Charles H. Nelson ${ }^{3}$ \\ ${ }^{1}$ Stockholm Observatory, Department of Astronomy, Stockholm University, AlbaNova, 10691 Stockholm, Sweden \\ ${ }^{2}$ Oskar Klein Center for Cosmoparticle Physics, Stockholm University, 10691 Stockholm, Sweden \\ ${ }^{3}$ Physics and Astronomy Department, Drake University, 2507 University Avenue, Des Moines, IA 50311, USA \\ Received 2010 March 3; accepted 2010 August 29; published 2010 October 11
}

\begin{abstract}
Nuclear regions of galaxies generally host a mixture of components with different excitations, compositions, and kinematics. Derivation of emission line ratios and kinematics could then be misleading if due correction is not made for the limited spatial and spectral resolutions of the observations. The aim of this paper is to demonstrate, with application to a long-slit spectrum of the Seyfert 2 galaxy NGC 1358, how line intensities and velocities, together with modeling and knowledge of the point-spread function, may be used to resolve the differing structures. In the situation outlined above, the observed kinematics differs for different spectral lines. From the observed intensity and velocity distributions of a number of spectral lines, and with some reasonable assumptions about the structure of different subcomponents to diminish the number of free parameters, the true line ratios and velocity structures may be deduced. A preliminary solution for the nuclear structure of NGC 1358 is obtained, involving a nuclear point source and an emerging outflow of high excitation, ending with shock and postshock cloud as revealed by the velocities, as well as a nuclear emission-line disk rotating in the potential of a stellar bulge and expressing a radial excitation gradient. The method results in a likely scenario for the nuclear structure of the Seyfert 2 galaxy NGC 1358. For definitive results, an extrapolation of the method to two dimensions combined with the use of integral field spectroscopy will be necessary.
\end{abstract}

Key words: galaxies: active - galaxies: kinematics and dynamics - galaxies: structure - methods: data analysis

Online-only material: color figures

\section{INTRODUCTION}

The nuclear and circumnuclear activity of galaxies generally involves the interplay among a number of different components and phenomena, e.g., a central active source surrounded by an absorbing torus, a rotating central bulge, outflowing jets, flowing streams due to the action of a bar, or even merging. To separate these different components and to derive their respective line ratios and kinematic behavior are generally difficult due to the limited spatial and spectroscopic resolution available. On the other hand, such a separation is crucial to the analysis of the structures and physical processes involved in the nuclear region and their roles in galaxy evolution.

Then, as to be demonstrated here, such a separation could benefit by considering the differences of the velocities observed for different spectral lines and can be eased by models of the activity, smoothed with the point-spread function (PSF) and fitted to the observations.

NGC 1358 (Figure 1) is a barred Sa galaxy hosting an active galactic nucleus (AGN) at a heliocentric velocity of $4028 \mathrm{~km} \mathrm{~s}^{-1}$ (Theureau et al. 1998), which is included in the distancelimited sample of Ulvestad \& Wilson (1989) as a Seyfert 2. It attracted our attention because of its remarkable circumnuclear kinematics which also allows the demonstration of the utility of the method introduced here.

Assuming a Hubble constant of $71 \mathrm{~km} \mathrm{~s}^{-1} \mathrm{Mpc}^{-1}$ (Spergel et al. 2003), the distance of NGC 1358 amounts to $57 \mathrm{Mpc}$. Then, $1^{\prime \prime}$ corresponds to $275 \mathrm{pc}$. The outer optical disk of NGC 1358, as seen in Figure 1, including the narrow spiral arms, has a radius of $1^{\prime}$, or $16.5 \mathrm{kpc}$. The bar along a position angle (P.A.)

\footnotetext{
* Based on observations at the European Southern Observatory.
}

of $130^{\circ}$ has a semimajor axis length of $19^{\prime \prime}$ (Gerssen et al. 2003) or $5.2 \mathrm{kpc}$.

The continuum peak at the nucleus shows a half-power halfwidth of 1".3. Dumas et al. (2007) derived, by integral field spectroscopy, the velocity fields of the ionized gas in the central regions in the emission line of [O III] $\lambda 5007$ as well as in the stellar absorption lines (the latter displayed only in a poster). The stellar velocity field shows a kinematic major axis in a P.A. of 11.5 with the north side receding. This is also close to the assumed P.A. of the line of nodes of the outer disk. The authors derive a symmetric stellar rotation curve out to $\pm 4^{\prime \prime}$ from the nucleus. The [O III] velocity field, on the other hand, shows a kinematic major axis in about P.A. $90^{\circ}$ with the east side receding. Narrowband imaging in $[\mathrm{O}$ III $] \lambda 5007$ shows a slightly $S$-shaped emission-line region with a maximum intensity 1.'6 from the nucleus, again along a P.A. of about $130^{\circ}$ (Dumas et al. 2007). This feature shows clearly deviating negative velocities. Dumas et al. (2007) suggest that the elongated nuclear emissionline region corresponds to gaseous streaming due to an inner bar inside the inner Lindblad resonance of the outer bar.

A Hubble Space Telescope/Space Telescope Imaging Spectrograph (HST/STIS) spectrum covering the [S II] $\lambda \lambda$ 6716, 6731 lines, within an aperture of size $0^{\prime} .2$ centered at the nucleus, shows double components with the fainter component blueshifted with respect to the stronger (Rice et al. 2006).

\section{OBSERVATIONS}

We have observed NGC 1358 with the EFOSC2 spectrograph on the ESO $2.2 \mathrm{~m}$ telescope and obtained a long-slit spectrum over the nucleus in P.A. $85^{\circ}$ on 1996 December 6. With this P.A. a star was brought on the slit, which gave a check on the P.A. as 


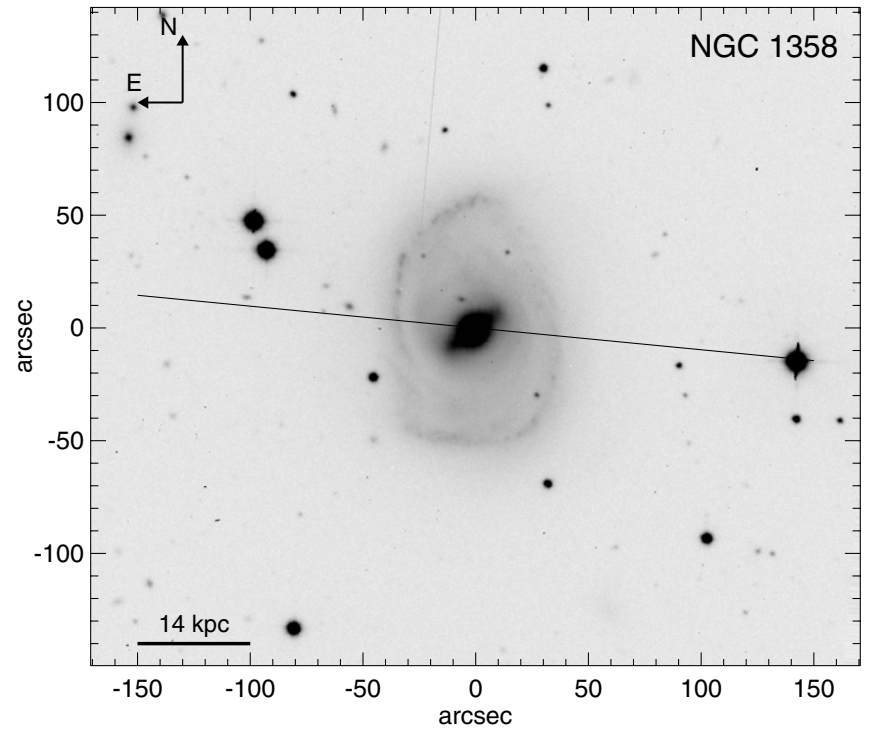

Figure 1. NGC 1358 optical image obtained with the EFOSC2 spectrograph on the ESO $2.2 \mathrm{~m}$ telescope. The straight line indicates the position of the slit (P.A. $85^{\circ}$ ) for the spectrum discussed in this paper. The slit crosses the spiral arms at about $35^{\prime \prime}$ galactocentric distance.

well as the PSF. The slit cuts the main spiral arms about $35^{\prime \prime}$ east and $35^{\prime \prime}$ west of the nucleus (Figure 1). The spectrum covered the wavelength region $4075-7000 \AA$, where the strongest spectral features are the $\mathrm{H} \beta \lambda$ 4861, [O III] $\lambda \lambda$ 4960, 5007, $\mathrm{H} \alpha \lambda$ 6563, [N II] $\lambda \lambda$ 6548, 6584, and [S II] $\lambda \lambda$ 6716, 6731 emission lines and the $\mathrm{Mgb}$ and $\mathrm{NaD}$ absorption lines (Figure 2). In the spatial direction, the pixel size corresponds to 0 '.26. For flux calibration, the star Hiltner 600 was chosen.

Figure 2 shows two fully reduced and flux-calibrated example spectra obtained from the long-slit spectrum. To derive the distribution and kinematic parameters, we have fitted the galaxy spectrum in two different ways: first, by using the standard IRAF routine SPLOT to fit Gaussians to the emission and absorption lines, and second, by using the simultaneous gas and absorption line fitting interface description language (IDL) routine developed by Sarzi et al. (2006) and Falcón-Barroso et al. (2006). The latter method is designed to separate the relative contributions of stellar continuum and absorption features and nebular emission in spectra of galaxies while measuring the gas emission and kinematics. Accordingly, it uses the routine of Cappellari \& Emsellem (2004) to find the bestfitted linear combination of single stellar population spectra by Vazdekis (1999) to build a stellar spectrum representative of the observed galaxy spectrum for this wavelength range and with the same spectral resolution. Simultaneously, the nebular emission lines have been fitted, and the results yield the gas and stellar kinematics. We found that both routines deliver fully comparable gas distribution and kinematics, with the advantage that the IDL routine has derived a much more robust stellar kinematics.
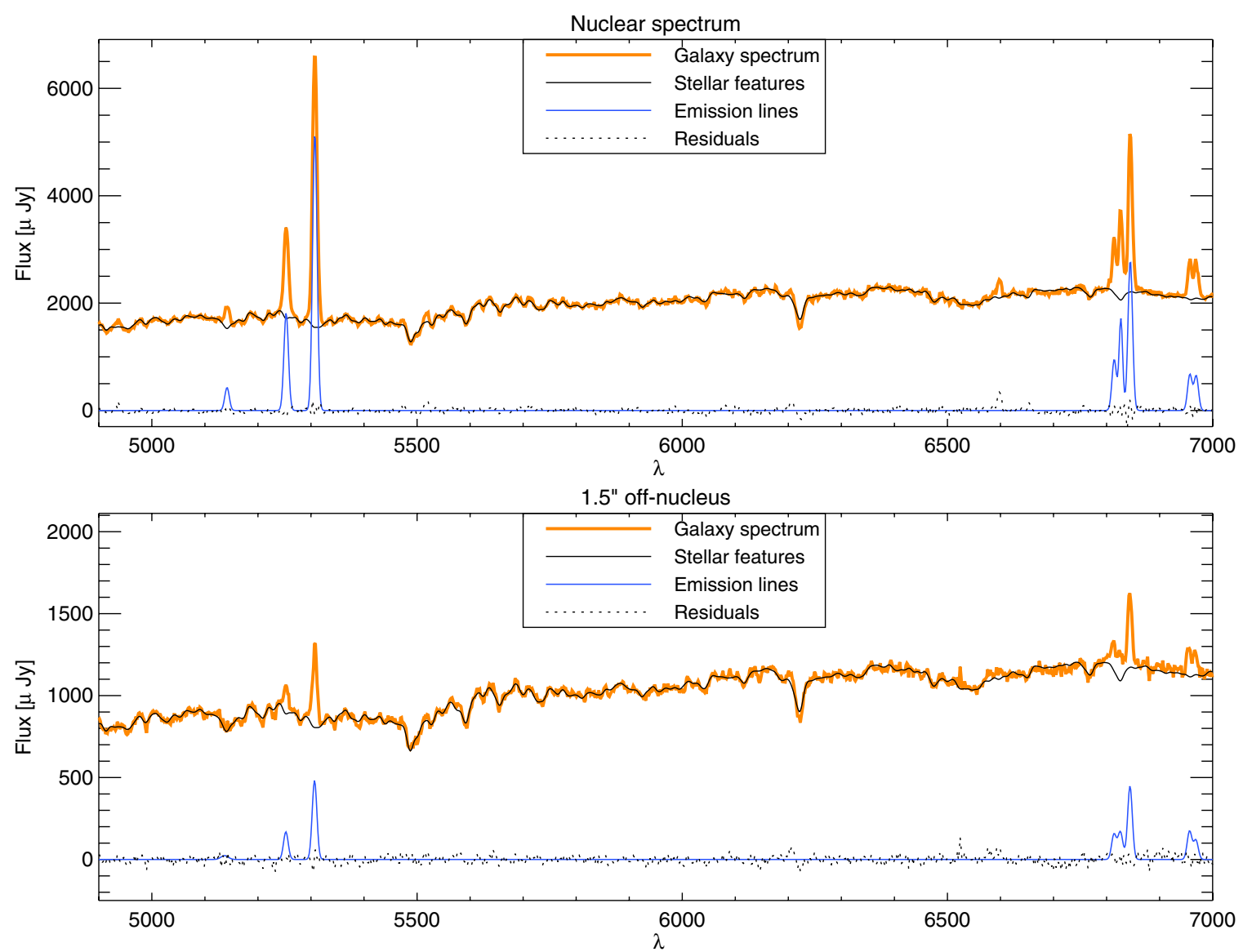

Figure 2. Spectrum of the nucleus (top) and 1".5 west of the off-nucleus region of NGC 1358. The best-fitted linear combination of single stellar populations shows the stellar features fitted simultaneously as Gaussian fits to the emission lines have been applied. The residuals (data - total fit) show that the fits have been successful ([O I] $\lambda \lambda$ 6300, 6364 have been excluded in this fit).

(A color version of this figure is available in the online journal.) 


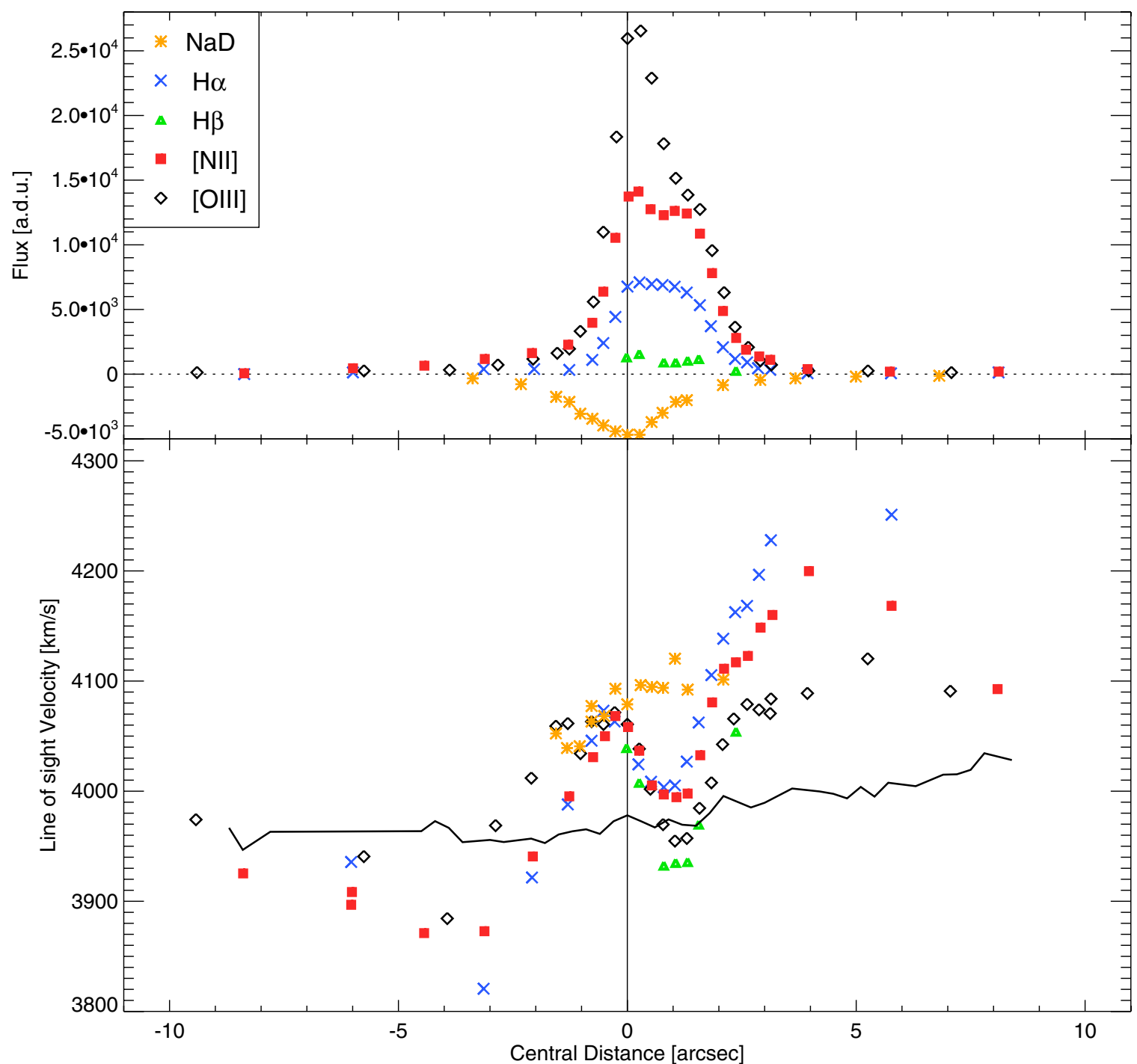

Figure 3. Fluxes and velocities over the central region of NGC 1358 along the slit in P.A. $85^{\circ}$. The flux scale is arbitrary. The solid curve illustrates the velocity of the stellar component as derived from all the stellar features present in the spectra. East is to the right and the zero point of the abscissa corresponds to the maximum of the continuum (Figure 5), and the NaD absorption line flux values have been inverted for presentation purposes. We have derived velocity uncertainties of the order of $10 \mathrm{~km} \mathrm{~s}^{-1}$.

(A color version of this figure is available in the online journal.)

Figure 3 shows the run of $[\mathrm{N}$ II] $\lambda$ 6584, $\mathrm{H} \alpha,[\mathrm{O}$ III $] \lambda$ 5007, and $\mathrm{H} \beta$ emission as well as the $\mathrm{NaD}$ absorption intensities and velocities over the central region of NGC 1358 along the slit as derived with the SPLOT routine. The solid line shows the velocities of the stellar component derived from all the stellar features. As the zero point of the abscissa, we have chosen the position of the maximum of the continuum (cf. Figure 5). In Figure 3 the emission-line velocities, and in particular $\mathrm{H} \alpha$ and [N II], show a rapidly increasing rotation curve with a maximum of about 4" from the center. Dumas et al. (2007) show that this nuclear emission-line disk should have a kinematic major axis directed close to P.A. $90^{\circ}$, i.e., only $5^{\circ}$ from the P.A. of the present slit position, but almost perpendicular to the kinematic major axis of the stellar bulge.

Within 2" from the center, on the east side, we see a reversal, or a dip, in the apparent rotation curve of the emission lines. In the $\mathrm{NaD}$ absorption line velocities, this dip is absent. From Figure 3, it is clear that the emission-line velocity dip corresponds to an excess of the emission-line intensities up to $2^{\prime \prime}$ from the nucleus. The $\mathrm{NaD}$ lines show no such asymmetry and the position of the maximum absorption coincides with that of the maximum of continuum emission.

The simplest interpretation of the gas kinematics observed in Figure 3 seems to be a central point source and a jet-like outflow that are superimposed on a gaseous disk rotating in the potential of the stellar bulge. Preliminary simulations along the scheme presented below suggested that the jet outflow line-ofsight velocities should be of the order of $-200 \mathrm{~km} \mathrm{~s}^{-1}$.

Support for this scenario is found in the [S II] emissionline profiles from the HST/STIS spectra published by Rice et al. (2006) reproduced in Figure 4. One spectrum is observed through a $1^{\prime \prime} .0$ aperture and another through an aperture of 0.2 . The applied Gaussian fits have been constrained to enforce the relative separation between the [S II] doublets. In both spectra, a strong component is seen in the two lines with very similar flux ratios in the two apertures. In the $0 ! 2$ aperture spectrum, a secondary blueshifted component is seen. We have assumed $10,000 \mathrm{~K}$ electron temperature and used the atomic parameters of Mendoza (1983) and Osterbrock (1989) to calculate the 

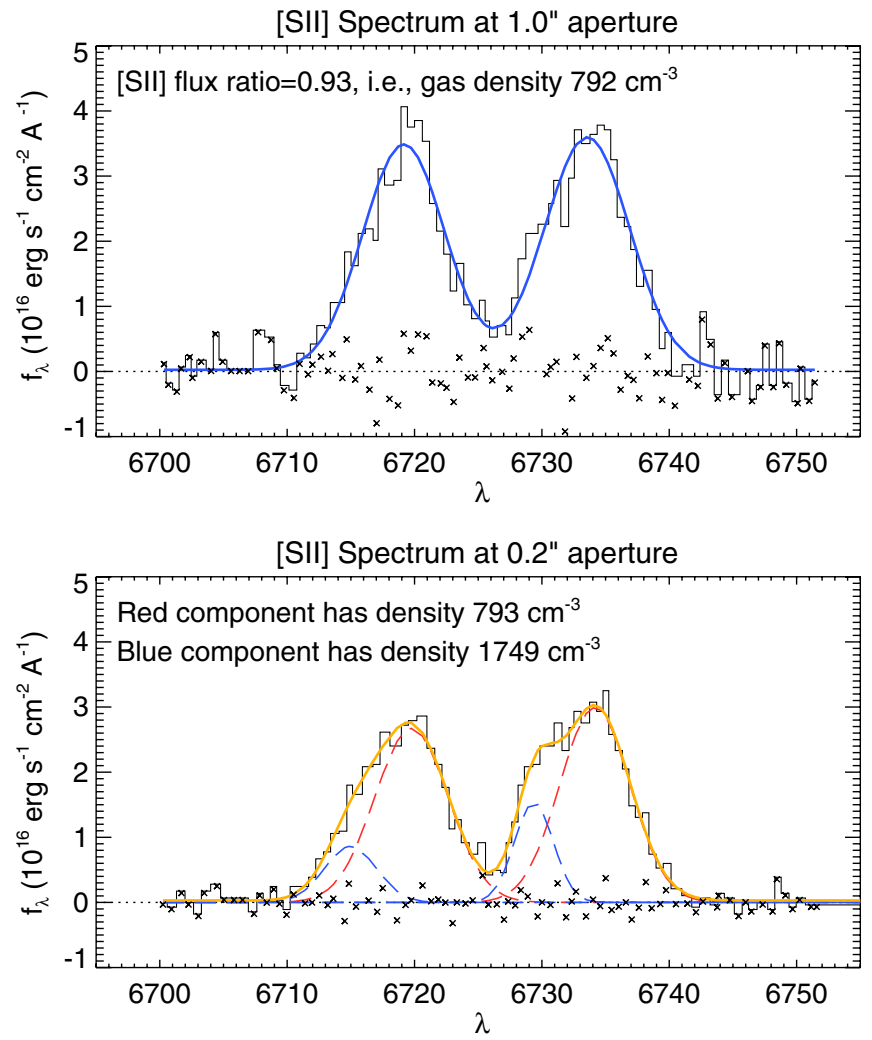

Figure 4. [S II] $\lambda \lambda$ 6716, 6731 spectrum from HST/STIS observations of the nucleus of NGC 1358 (Rice et al. 2006), with constrained Gaussian fits. In the bottom panel, the two components are separated by $214 \mathrm{~km} \mathrm{~s}^{-1}$, and all densities are calculated assuming 10,000 K electron temperature. Unconstrained Gaussian fits result in almost identical fits.

(A color version of this figure is available in the online journal.)

electron density from these spectra. The calculated relative fluxes yield for the spatially extended and dominating component an electron density of $793 \mathrm{~cm}^{-3}$. The central component, only appearing in the 0.2 aperture spectrum, has a density of $1749 \mathrm{~cm}^{-3}$. Being the innermost part of the jet, this denser component is fainter and blueshifted with a velocity difference between the strong and faint components of $214 \mathrm{~km} \mathrm{~s}^{-1}$, respectively.

The stellar velocities show only a very slight variation over the region in agreement with the slight positive gradient expected for the stellar velocity field of Dumas et al. (2007) with the present slit inclined $74^{\circ}$ to the stellar kinematic major axis. The $\mathrm{NaD}$ absorption in front of the nucleus appears to have a higher redshift than the average stellar template redshift, which is also indicated by the shape of the line residuals in Figure 2.

Most remarkably, the velocity dip to the east of the nucleus is larger for [O III] than for [N $\mathrm{II}]$ and $\mathrm{H} \alpha$ lines are apparently still larger for $\mathrm{H} \beta$. Outside the dip, [O III] shows smaller rotational velocities than $\mathrm{H} \alpha$ and [N II]. Here we ask ourselves: what additional information on the structure and ionization distribution in the nuclear region do these differences in velocity behavior give us?

\section{THEORY}

An explanation for the differences in velocity behavior between the various emission lines would be that the whole central region consists of different gaseous components with different velocity behaviors, excitations, and dust absorptions, and thus different sets of line ratios. When this region is smoothed by the PSF, different distortions of the velocity field for the different lines will result.

Assume that the region considered covers $N$ pixels and contains $K$ separate structural components, each with its own line ratios and set of velocities. If $J_{i}^{(\lambda)}$ and $V_{i}^{(\lambda)}$ are the observed line intensities and velocities at a pixel number $i$ of a line with the wavelength $\lambda$, we then have

$$
\begin{gathered}
J_{i}^{(\lambda)}=\sum_{j} \sum_{k} I_{k j}^{(\lambda)} p(i-j), \\
V_{i}^{(\lambda)}=\frac{1}{J_{i}^{(\lambda)}} \sum_{j} \sum_{k} v_{k j} I_{k j}^{(\lambda)} p(i-j),
\end{gathered}
$$

where $I_{k j}^{(\lambda)}$ is the intrinsic intensity, in the line $\lambda$, of the component $k=(1, \ldots, K)$ at the pixel $j, v_{k j}$ is the corresponding velocity, which is supposed to be independent of $\lambda$, and $p(x)$ is the PSF. If $L$ is the number of lines measured, we have in the general case $K N(L+1)$ unknown and $2 N L$ equations. If the line ratios are all the same in the different components, Equation (2) is independent of $\lambda$. In the simplest case, where we only consider one component $(K=1)$, we have enough equations to solve for the unknown intensities and velocities for each $\lambda$, which can be made by CLEAN-like algorithms (Högbom 1974).

In the general case, we do not have a sufficient number of equations to solve for the unknown intensities $I_{k j}^{(\lambda)}$ and velocities $v_{k j}$. If, however, we increase the number of lines measured and at the same time impose some conditions on the unknowns in terms of models, we may in principle be able to reach a solution. For instance, we see from Equations (1) and (2) that if the line ratios $I_{k j}^{\left(\lambda_{1}\right)} / I_{k j}^{\left(\lambda_{2}\right)}$ depend on $k$, i.e., they do vary from one component to another, then we have $V_{i}^{\left(\lambda_{1}\right)}-V_{i}^{\left(\lambda_{2}\right)} \neq 0$. Thus, the differences between velocities from different spectral lines give us information about differing line ratios between different components. We will illustrate this for our spectrum for NGC 1358.

\section{MODELS}

We will test velocity and intensity observations over the nuclear region against a scenario where the dip in the rotation curve is caused by an outflow of the excited gas from a central source at rest with respect to a nuclear gaseous disk. We strive to have in this model an absolute minimum of free parameters to fit the observations. We will first demonstrate the simplest primitive model, here called Model 1, to explain our motivation for introducing our more elaborate models.

\subsection{Model 1}

Our Model 1 has the following features.

1. A stellar bulge with a mass distribution corresponding to the continuum surface intensity distribution, giving the gravitational field in the nuclear region. As seen in Figure 5, the observed surface intensity distribution can be well represented by the sum of two exponential disks with scale lengths of $0{ }^{\prime} 7$ and $4^{\prime \prime}$, respectively, and an intensity ratio of 4:1, smoothed with the observed PSF. To derive a likely potential and rotation curve for this stellar bulge, we use relations (2)-(170) of Binney \& Tremaine (1987) to give the potential of the sum of two exponential spheres 


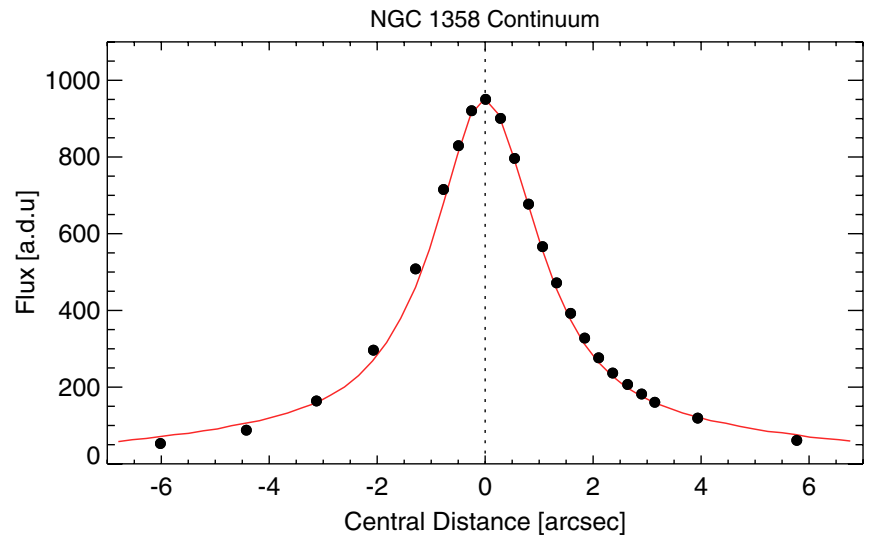

Figure 5. Continuum levels under the emission-line $\mathrm{H} \alpha$ fitted with the sum of two exponential disks of scale lengths 0.7 and $4^{\prime \prime}$ and the central intensity ratio 4/1, smoothed with the PSF.

(A color version of this figure is available in the online journal.)

with scale lengths 0.7 and $4 . .0$, respectively, and a mass ratio of 4:1 in conformance with Figure 5. Although we note that routinely bulges are fitted by de Vaucouleurs or Sersic profiles, experience has shown that exponential profiles are satisfactory in parameterizing the bulge (e.g., Fathi \& Peletier 2003, Balcells et al. 2007), and certainly this also seems to be the case for NGC 1358. This makes the total mass $M \times \sin ^{2}(i)$ of the bulge the only free parameter of the bulge potential, where $i$ is the inclination of the plane of the emission-line disk to the plane of the sky, and we assume that the difference in P.A. between the slit and the line of nodes of the disk is small.

2 . In the potential of this bulge, there is a rotating emissionline disk. To avoid edge problems, we assume this disk to be exponential and rotate with circular velocities. As the intensities of the disk is low compared to the other features, the exact scale length is not crucial, and we fix it ad hoc to 4 .' 0 . Then the only free parameters are the central surface line intensities of the emission-line disk.

3. A central emission-line point source coinciding with the center of the bulge and the emission-line disk and at rest with respect to that center.

4. A gas flow, here called the "jet," seen from the nucleus out to 7 pixels (1".8) from the nucleus with constant line-of-sight velocity $-214 \mathrm{~km} \mathrm{~s}^{-1}$ with respect to the point source, which was the velocity of the fainter component relative to the stronger one as seen in the HST/STIS spectrum (Figure 4).

The resulting intensities and velocities are convolved with the observed PSF as derived from a field star on the slit (Figure 6).

The line-of-sight velocity, or redshift, of the central source and disk is an open question. Theureau et al. (1998) report a redshift of $4028 \mathrm{~km} \mathrm{~s}^{-1}$, while Dumas et al. (2007) estimate the systemic redshift of the galaxy at $3997 \pm 13 \mathrm{~km} \mathrm{~s}^{-1}$. Rice et al. (2006) assume a systematic redshift of $4008 \mathrm{~km} \mathrm{~s}^{-1}$, and their spectrum (Figure 4) shows a line-of-sight velocity of $+145 \mathrm{~km} \mathrm{~s}^{-1}$ for the stronger nuclear component with respect to the $4008 \mathrm{~km} \mathrm{~s}^{-1}$ systemic velocity, i.e., a nuclear redshift of $4153 \mathrm{~km} \mathrm{~s}^{-1}$ for the stronger component and $3939 \mathrm{~km} \mathrm{~s}^{-1}$ for the blueshifted weaker component. We prefer to make the systemic velocity of the nuclear disk and the central emission source a free parameter in our model. The additional free parameters are the intensities of the central source along the jet for each emission line.

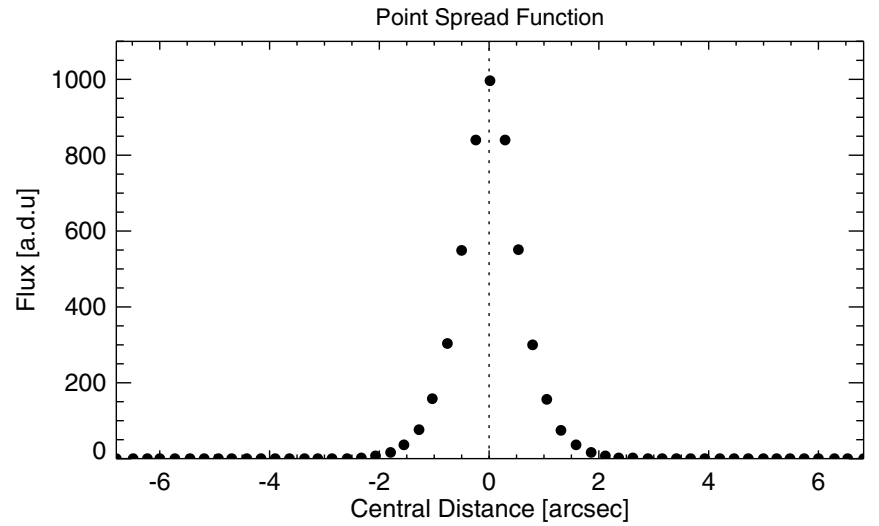

Figure 6. PSF as derived from the star $150^{\prime \prime}$ west of NGC 1358 .

The fit of this preliminary model is given in Figure 10. As can be seen, this model cannot reproduce a very sharp rise in the emission-line velocity curves at the end of the jet about $2^{\prime \prime}$ to the east of the center. Thus, we feel forced to introduce a sharp shock at the end of the jet and a postshock cloud.

\subsection{Model 2}

This model has the following features.

1. A stellar bulge identical with the bulge in Model 1 presented above, where the total mass $M \times \sin ^{2}(i)$ of the bulge is the only free parameter.

2. In the potential of this bulge, a rotating exponential emission-line disk similar to the one in Model 1 again with a scale length of 4".0 and the only free parameters are the central surface line intensities.

3. A central emission-line point source coinciding with the centers of the bulge and the emission-line disk and at rest with respect to that center.

4. A gas flow, here called the "jet," seen from the nucleus out to a certain number of pixels from the nucleus, with constant line-of-sight velocity $-214 \mathrm{~km} \mathrm{~s}^{-1}$ with respect to the point source. This again is the velocity of the fainter component relative to the stronger one as seen in the HST/STIS spectrum (Figure 4). In a chosen pixel at the end of the jet, we introduce a sharp shock, with a jump to more positive velocities, and in the following pixels, up to pixel 8 , a postshock cloud with constant velocity. The mean velocity in the shock pixel is given some intermediate velocity between those of the jet and the postshock cloud.

The resulting intensities and velocities are convolved with the observed PSF as derived from a field star on the slit (Figure 6).

Still, in this way we have tried to keep the free parameters to a minimum, and the free parameters of this model, to be fitted to the observations, are

1. the total mass $M \times \sin ^{2}(i)$ of the bulge;

2. the common redshift of the central point source and the center of the emission-line disk;

3. the intensity of the central point source for each emission line;

4. the central intensity of the emission-line disk for each emission line;

5. the pixel to contain the shock and the intensity and velocity in that pixel;

6. the intensities along the jet flow for each emission line; and 


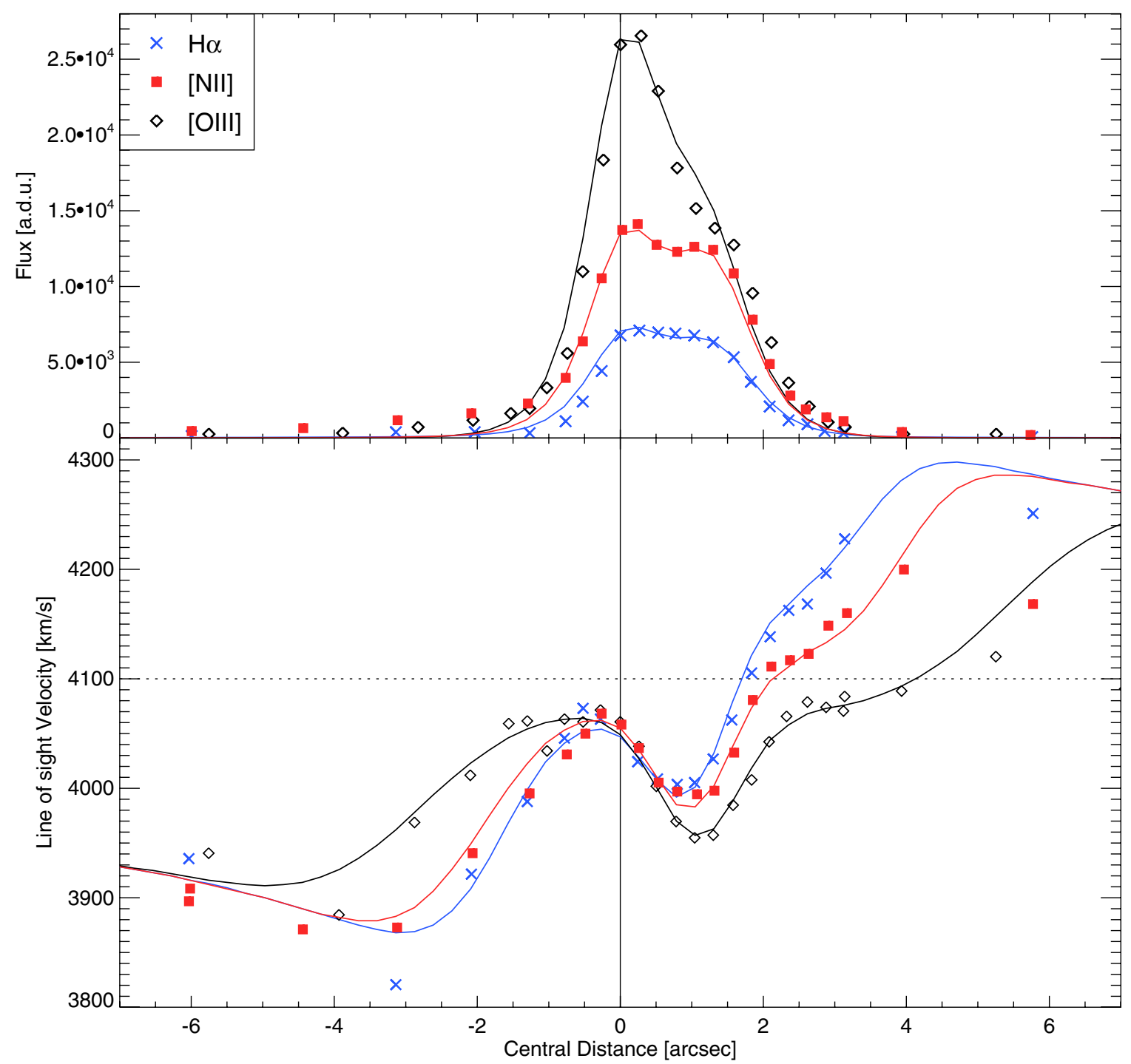

Figure 7. Best-fit model to the emission-line intensities and line-of-sight velocities (Model 3 as described in the text). The dotted line indicates the derived redshift of the nuclear source.

(A color version of this figure is available in the online journal.)

7. the common velocity and the intensities in the postshock cloud for each emission line.

These parameters are varied until a reasonable fit to the observed velocities and intensities have been obtained. The fit is displayed in Figure 11. The shock is placed within pixel 5 with the intermediate velocity of $-100 \mathrm{~km} \mathrm{~s}^{-1}$. This determines the postshock cloud velocity to about $+100 \mathrm{~km} \mathrm{~s}^{-1}$. As can be seen in Figure 11, the line intensities of [O III] and the velocities in the jet are not perfectly fitted, and to place the shock short of pixel 5 makes the situation worse.

Furthermore, it can be seen from the observations that the emission lines have long faint wings on the western (negative) side, actually $\mathrm{H} \alpha$ and $[\mathrm{N}$ II] stretching furthest and [O III] having the shortest wing. The run of the $\mathrm{H} \alpha$ and [N II] velocities on the west side show that the main intensities of this extended emission come from the rotating disk. This indicates an asymmetry in the emission-line disk, and this makes it difficult with the present model to fit the velocities away from the center simultaneously on both sides, as can be seen in Figure 11. Due to the jet, the observed velocities on the left side are more influenced by the inner part of the disk and on the right side by the outer part.
We can then introduce an asymmetry of the observations without abandoning the circular symmetry of the disk by describing its surface intensity as the sum of two superposed exponential disks with widely different scale lengths and varying their central intensities independently. This results in differences in the line ratios, simulating a gradient of these ratios within the disk. This leads us to the next model.

\subsection{Model 3}

This model is the final one which gives us the best fit to the observed velocities and intensities in the nuclear region of the galaxy. Its features are as follows.

1. A stellar bulge identical with the bulge in Model 1 presented above, where the total mass $M \times \sin ^{2}(i)$ of the bulge is the only free parameter.

2. In the potential of this bulge there is a rotating emissionline disk. We assume the intensities in the disk to be the sum of two exponential disks with differing scale lengths. As the intensities of the disk are low compared to the other features, the exact scale lengths are not crucial, and we fix 

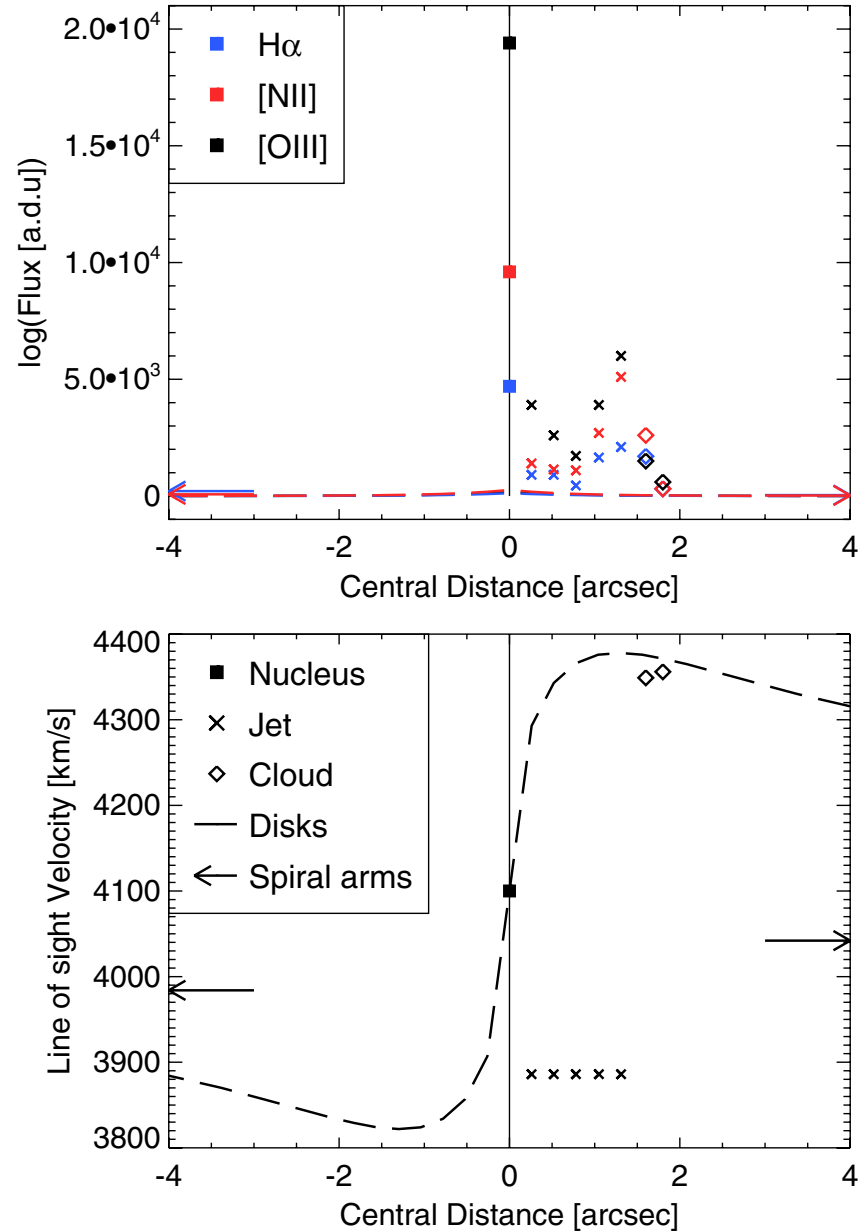

Figure 8. Illustrating fluxes and velocities of the individual components listed in Table 1 without the effect of the PSF. The arrows show the fluxes and velocities assigned to the spiral arms which are fixed at $-37^{\prime \prime}$ and $33^{\prime \prime}$ galactocentric distance. Filled boxes illustrate the nucleus, crosses the jet, and diamonds the cloud, respectively.

(A color version of this figure is available in the online journal.)

them ad hoc to 0.7 and $4 . \prime 0$ (the same as for the bulge only for historical reasons). Then the only free parameters are the central surface line intensities of the two disks (here called Disk 1 and Disk 2) representing the inner and outer parts, respectively, of the emission-line disk.

3. A central emission-line point source coinciding with the centers of the bulge and the emission-line disk and at rest with respect to that center.

4. A gas flow, here called the "jet," seen from the nucleus out to a certain number of pixels from the nucleus, with constant line-of-sight velocity $-214 \mathrm{~km} \mathrm{~s}^{-1}$ with respect to the point source. This again is the velocity of the fainter component relative to the stronger as seen in the HST/STIS spectrum (Figure 4). At the end of this jet, at the border between two pixels, we introduce a sharp shock, with a jump to more positive velocities, and in the following pixels, up to pixel 7, a postshock cloud with constant velocity.

The resulting intensities and velocities are convolved with the observed PSF as derived from the field star on the slit (Figure 6).

The number of free parameters of this model is less than that of Model 2 and they are as follows.

1. The total mass $M \times \sin ^{2}(i)$ of the bulge.

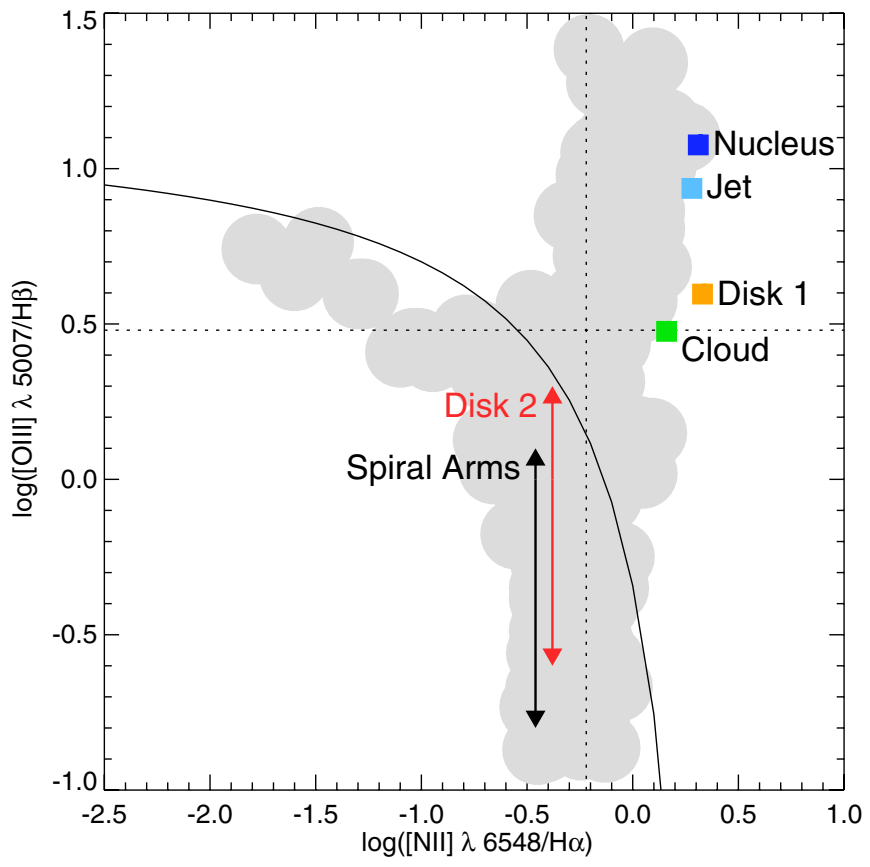

Figure 9. Line ratio diagnostics derived from our ESO spectra. The solid curve shows the starburst-AGN separation lines of Kewley et al. (2001), with the shaded region indicating their sample galaxies.

(A color version of this figure is available in the online journal.)

2. The common redshift of the central point source and the center of the emission-line disk.

3. The intensity of the central point source for each emission line.

4. The central intensities of the two superimposed emission disks for each emission line.

5. The position of the last pixel of the jet.

6. The intensities along the jet flow for each emission line.

7. The common velocity and the intensities in the postshock cloud for each emission line.

The fit of Model 3 to the velocities and intensities of the various emission lines is displayed in Figure 7, and the parameters are given in Table 1. The table also contains measurements for the spiral arms with the SPLOT routine where they intersect with the present slit.

\section{RESULTS}

Our best fit, Model 3, gives an observed redshift of the central source of $4100 \mathrm{~km} \mathrm{~s}^{-1}$, or $4089 \mathrm{~km} \mathrm{~s}^{-1}$ with heliocentric velocity correction. The total $M \times \sin ^{2}(i)$ of the bulge is determined essentially by the velocities of $\mathrm{H} \alpha$ and $\left[\mathrm{N}_{\mathrm{II}}\right]$ at the distance $-6^{\prime \prime}$ from the center. The best fit gives $M \times \sin ^{2}(i)=$ $14 \times 10^{9} M_{\odot}$. The remaining parameters of this fit are given in Table 1. Figure 8 illustrates Table 1 and gives the intensities and velocities of the various features before smearing with the PSF.

The central intensities of the emission-line disks are essentially given by the run of velocities from $-3^{\prime \prime}$ to $-1^{\prime \prime}$ and from $+2^{\prime \prime}$ to $+4^{\prime \prime}$ from the center.

The position of the shock falls at the border between pixels 5 and 6 , i.e., 1 1.5, from the center. This, in turn, forces the postshock velocity up to about $+250 \mathrm{~km} \mathrm{~s}^{-1}$ in pixels 6 and 7 .

Figure 7 shows that the velocities and intensities can be reproduced with fair accuracy within this nuclear region. Finally, the diagnostic line ratios $\log \left[\mathrm{N}_{\mathrm{II}}\right] / \mathrm{H} \alpha$ and $\log \left[\mathrm{O}_{\mathrm{III}}\right] / \mathrm{H} \alpha$ are 
Table 1

Parameters for the Model Presented in Figure 7

\begin{tabular}{|c|c|c|c|c|c|c|c|c|c|c|c|c|}
\hline \multirow{2}{*}{$\frac{\text { Component }}{\text { Pixel }}$} & \multirow{2}{*}{$\begin{array}{c}\text { Nucleus } \\
0\end{array}$} & \multicolumn{5}{|c|}{$\longleftarrow$ Jet $\longrightarrow$} & \multicolumn{2}{|c|}{$\longleftarrow$ Cloud $\longrightarrow$} & \multirow[t]{2}{*}{ Disk 1} & \multirow[t]{2}{*}{ Disk 2} & \multicolumn{2}{|c|}{$\longleftarrow$ Spiral Arms $\longrightarrow$} \\
\hline & & 1 & 2 & 3 & 4 & 5 & 6 & 7 & & & 128 & -140 \\
\hline Arcsec & 0 & 0.26 & 0.52 & 0.78 & 1.05 & 1.31 & 1.6 & 1.8 & $r_{d}=0.7$ & $r_{d}=4$ & 33 & -37 \\
\hline Velocity $\left(\mathrm{km} \mathrm{s}^{-1}\right)$ & 0 & -214 & -214 & -214 & -214 & -214 & +250 & +250 & Rotating & Rotating & -58 & -116 \\
\hline Flux $(\mathrm{H} \alpha)$ & 4700 & 910 & 910 & 450 & 1650 & 2100 & 1700 & 310 & 116 & 29 & 210 & 40 \\
\hline Flux ([N II] $)$ & 9600 & 1400 & 1140 & 1100 & 2700 & 5100 & 2600 & 310 & 250 & 12 & 72 & 24 \\
\hline Flux ([O III $]$ ) & 19400 & 3900 & 2600 & 1720 & 3900 & 6000 & 1500 & 600 & 160 & 0 & & \\
\hline $\log ([\mathrm{N}$ II $] / \mathrm{H} \alpha)$ & 0.31 & & & $\longleftarrow 0.28 \longrightarrow$ & & & $\longleftarrow$ & $0.16 \longrightarrow$ & 0.33 & -0.38 & -0.46 & -0.22 \\
\hline $\log \left(\left[\mathrm{O}_{\mathrm{III}}\right] / \mathrm{H} \alpha\right)$ & 0.62 & & & $\longleftarrow 0.48 \longrightarrow$ & & & $\longleftarrow$ & $0.02 \longrightarrow$ & 0.14 & & & \\
\hline
\end{tabular}

Note. Disks 1 and 2 make up the emission-line disk with scale lengths $r_{d}$ of $0^{\prime \prime} 7$ and $4^{\prime \prime}$, respectively (cf. Figure 8). 


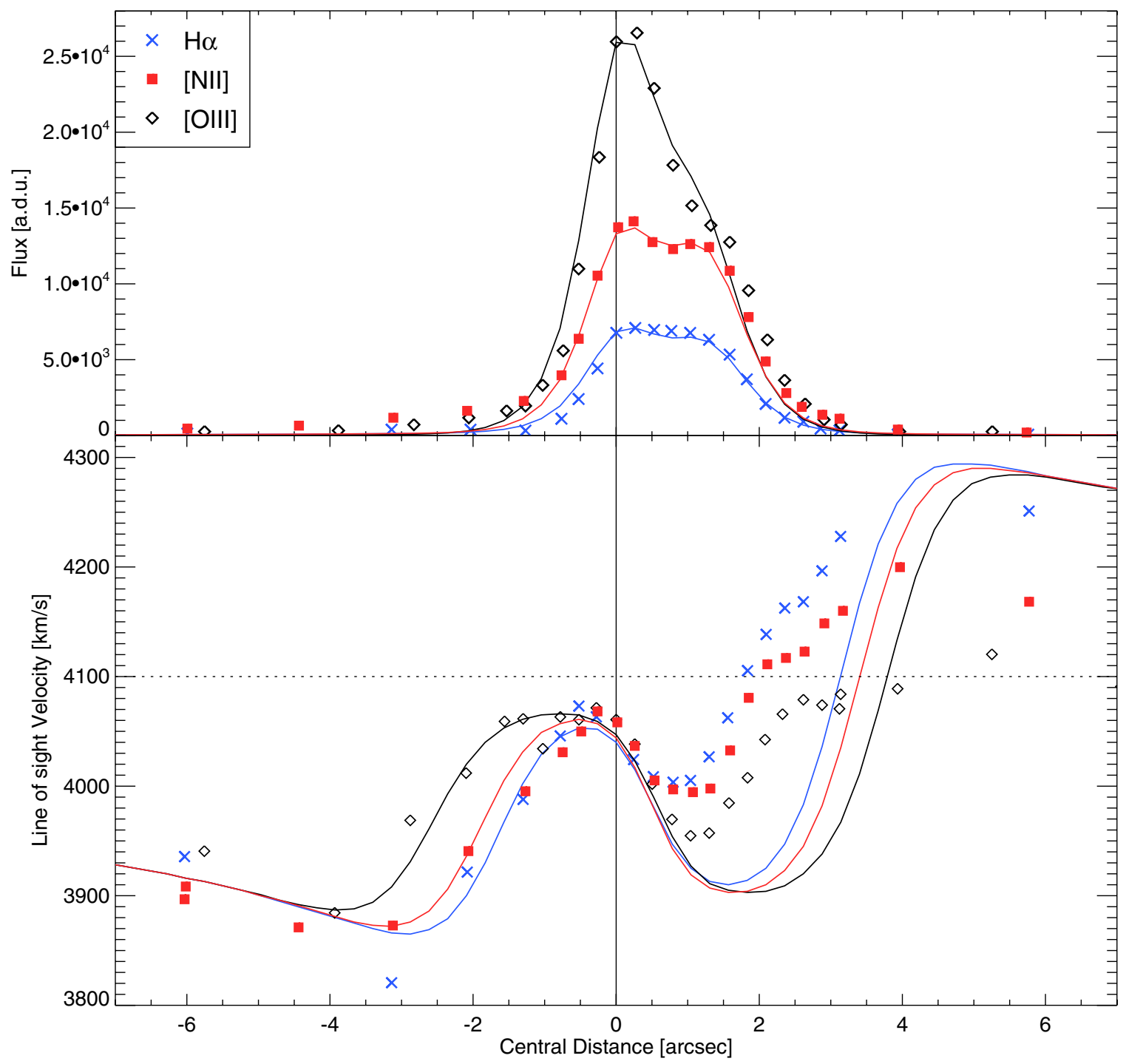

Figure 10. Fit of Model 1 to the observations.

(A color version of this figure is available in the online journal.)

given in Table 1 for various components. The $\mathrm{H} \beta$ intensities are too weak and uncertain to give a unique solution in the present case.

\section{DISCUSSION}

Our "best-fit" Model 3 resolves the nuclear structure of NGC 1358 into

1. a central unresolved emission-line source (the nucleus),

2. a 1".5 long jet emerging from the nucleus with a line-of-sight velocity of $-214 \mathrm{~km} \mathrm{~s}^{-1}$,

3. a spherical nuclear stellar bulge containing a rotating emission-line disk, inclined to the stellar kinematic symmetry plane of the bulge, and

4. an emission-line region, the postshock cloud, at the end of the jet, and after a drastic jump in velocity.

With the emission-line fluxes for these structures we are able to set up the starburst-AGN diagnostic diagrams introduced by Baldwin et al. (1981) and Veilleux \& Osterbrock (1987). Figure 9 shows the diagnostic diagram $\log [\mathrm{O}$ III $] / \mathrm{H} \beta$ versus $\log$ $[\mathrm{N} \mathrm{II}] / \mathrm{H} \alpha$ where the $[\mathrm{O} \mathrm{III}] / \mathrm{H} \beta$ ratio is derived from $[\mathrm{O} \mathrm{III}] / \mathrm{H} \alpha$ assuming large optical depth, referred to as Case B described in chapter 4.2 of Osterbrock (1989). Accordingly, for an electron temperature of $10,000 \mathrm{~K}$, the $\mathrm{H} \alpha / \mathrm{H} \beta$ is 2.87 , which we note is not much different from the case when the gas is assumed to be optically thin. As evident, the nuclear source and the jet fall in the region of highly excited AGNs, while the inner disk and the postshock cloud fall close to the region of Low Ionization Nuclear Emission line Regions. The ratio $[\mathrm{N} \mathrm{II}] / \mathrm{H} \alpha$ places the outer part of the nuclear disk and the spiral arms in the H II region domain.

The contribution from the underlying $\mathrm{H} \alpha$ and $\mathrm{H} \beta$ absorption lines only changes the derived emission-line ratios marginally. However, the effect of reddening has not been taken into account in the emission ratios presented in Figure 9 and Table 1. As we have used the unreddened ratio $\mathrm{H} \alpha / \mathrm{H} \beta$, our derived [O III] $/ \mathrm{H} \beta$ ratios presented in the diagram are lower limits in the presence of reddening. This is an effect that favors our conclusions about the nature of the components.

The nuclear point source shows the highest excitation. If $\mathrm{H} \beta$ would be included in this analysis, its derived true intensity in the nuclear point source with $\mathrm{H} \alpha$ would give the true absorption of the nuclear source of this Seyfert 2 galaxy. Unfortunately, 


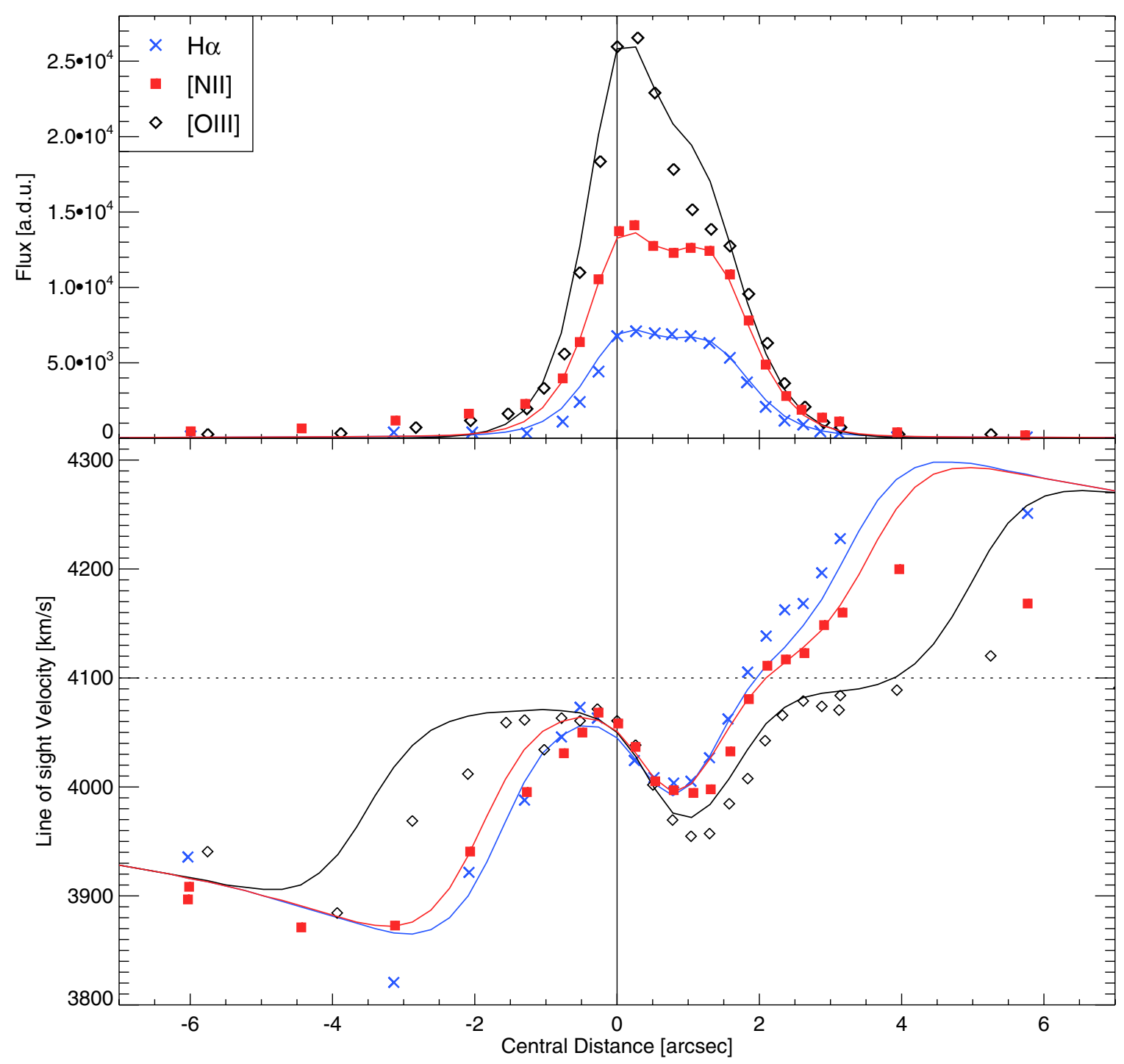

Figure 11. Fit of Model 2 to the observations.

(A color version of this figure is available in the online journal.)

$\mathrm{H} \beta$ is too faint and uncertain to be fitted with our procedure. Stronger exposed $\mathrm{H} \beta$ would be needed.

The emission-line map of Dumas et al. (2007) shows that the maximum intensity for our jet lies in about P.A. $130^{\circ}$, and there is also a fainter counterjet in the opposite direction. Thus, our slit avoids the maximum intensity of the jet and also the counterjet. As seen from Figure 7, this means that there is no need for a counterjet in our model. However, Disk 1, which is completely drowned on the east side by the jet, may in reality correspond to a trace of an absorbed counterjet. Its position in Figure 9 does not contradict this. On the other hand, the introduction of a counterjet in our model would add a number of uncertain intensity parameters.

The HST/STIS spectrum (Figure 4) indicates a high density for the jet, and the jet intensity maps display a bent spiral-like morphology consistent with a precessing jet flow. Considering the angle of about $45^{\circ}$ between the jet axis and our slit, the jet may be surrounded by a more widespread outflow, similar to the one observed in the nucleus of the Seyfert 1.5 galaxy NGC 1365 (Hjelm \& Lindblad 1996). Moran et al. (2000) conclude that NGC 1358 belongs to the class of about 50\% Seyfert 2s that do not contain a polarized broad-line region (NPBL Sy2). Shu et al. (2007) argue that NPBL Sy2s can be interpreted in the framework of the AGN unified model if the absorption column density is higher at large inclinations and the scattering region is absorbed at large inclinations. Thus, the inclination of the jet to the line of sight may be large and the outflow motion considerably higher than $214 \mathrm{~km} \mathrm{~s}^{-1}$.

The emission-line disk intensities are completely hidden behind the strong features in the central part and too faint to be measured at larger distances from the center. They are therefore completely determined by the influence of this disk on the observed total velocities. Disks 1 and 2 represent the inner and outer parts, respectively, of this nuclear emission-line disk. Their line ratios then indicate a rather strong gradient within the nuclear disk (see Table 1), or, as mentioned, a difference in line ratios between an eventual absorbed counterjet and Disk 2.

The disk rotates in the potential of the stellar bulge at an angle to the kinematic symmetry plane of the bulge. Our slit is oriented close to the kinematic major axis of the disk, but close to the kinematic minor axis of the stellar bulge (Dumas et al. 2007). The redshift of the central source in our model and the systematic redshift of the stellar bulge differ with about $100 \mathrm{~km} \mathrm{~s}^{-1}$ 
(Figure 3). The slit crosses the spiral arms close to the photometric minor axis of the outer galactic disk (Figure 1). Their mean velocity relative to the nucleus is $-87 \mathrm{~km} \mathrm{~s}^{-1}$. In view of the fact that our slit is parallel to the line of nodes of the emissionline velocity field and perpendicular to the line of nodes of the stellar velocity field, the difference in central velocity between our models and that of the stellar bulge, as seen in Figure 3, may be accounted for if our slit misses the central peak passing about $1^{\prime \prime}$ to the south of the nucleus. This would mean that our derived rotation curve is somewhat flatter than the true one and the intensities of the central source too faint. The $\mathrm{NaD}$ absorption lines, measured with SPLOT, show the same systematic redshift as the emission lines, which would imply that their main absorption comes from the emission-line disk and not from the stellar bulge. The negative mean velocity of the spiral arms speaks somewhat against this miscentering view with whatever weight this may have.

\section{CONCLUSIONS}

We want to stress that our method, i.e., because of the necessity to introduce the number of more or less reasonable assumptions in order to decrease the number of free parameters, does not give a unique solution to the nuclear structure of NGC 1358. The differing velocities in different lines give strong constraints for the line ratios. Experiments with our model, for example, show that it is the weakness of [O III] in the postshock cloud and the nuclear disk that causes the dip in the velocity of the eastern part of the velocity curve to be deeper in [O III], and the [O III] velocity curve to be flatter on both sides. But it is necessary to know with good precision the outer wings of the PSF in order to account for the influence of the strong components on the fainter ones.

In our particular case, the main drawback is that the observations are restricted to a single slit. To get all the necessary information about the velocities and spatial distribution of the different components from an isolated spectrum is futile because of the very limited coverage and the unknown influence due to the PSF from sources outside the slit. By adding more slits, there is still difficulty in obtaining an accurate and complete coverage (Lindblad et al. 1996). Obviously, the ideal is an integral field spectrometer and an extension of the method to a two-dimensional treatment, combined with comprehensive statistical analysis, covering the entire region of interest.

Nevertheless, we argue that, with this analysis, we have made a step toward the resolution of the nuclear region of NGC 1358 into a number of different components with different velocity behavior and excitation, where a summary of the results is seen in Figure 9 and Table 1.
We are indebted to the night assistants L. Ramirez and M. Turatto for their efficient assistance at the ESO telescope. IRAF is distributed by the National Optical Astronomy Observatory, which is operated by the Association of Universities for Research in Astronomy, Inc., under cooperative agreement with the National Science Foundation. K.F. acknowledges support from the Swedish Research Council (Vetenskapsrådet). Finally, we are grateful to an anonymous referee for valuable suggestions and remarks.

\section{APPENDIX}

\section{ALTERNATIVE MODELS}

The model presented in this paper has been obtained after a number of iterations fixing different free parameters. Here we show two models based on one single exponential emission-line disk, both reproducing the observations to different degrees of satisfaction (Figure 10 showing Model 1, and Figure 11 showing Model 2 as described in the text). Although the second model could be seen somewhat comparable to that shown in Figure 7, we chose Figure 7 as our preferred model since it reproduces most of the observed flux and kinematic features of NGC 1358.

\section{REFERENCES}

Balcells, M., Graham, A. W., \& Peletier, R. F. 2007, ApJ, 665, 1084

Baldwin, J. A., Phillips, M. M., \& Terlevich, R. 1981, PASP, 93, 5

Binney, J., \& Tremaine, S. 1987, Galactic Dynamics (Princeton, NJ: Princeton Univ. Press)

Cappellari, M., \& Emsellem, E. 2004, PASP, 116, 138

Dumas, G., Emsellem, E., \& Ferruit, P. 2007, ESO Astrophys. Symp., Science Perspectives for 3D Spectroscopy, ed. M. Kissler-Patig, J. R. Walsh, \& M. M. Roth (Berlin: Springer), 269

Falcón-Barroso, J., et al. 2006, MNRAS, 369, 529

Fathi, K., \& Peletier, R. F. 2003, A\&A, 407, 61

Gerssen, J., Kuiken, K., \& Merrifield, M. R. 2003, MNRAS, 345, 261

Hjelm, M., \& Lindblad, P. O. 1996, A\&A, 305, 727

Högbom, J. A. 1974, A\&AS, 15, 417

Kewley, L. J., Dopita, M. A., Sutherland, R. S., Heisler, C. A., \& Trevena, J. 2001, ApJ, 556, 121

Lindblad, P. O., Hjelm, M., Högbom, J., Jörsäter, S., Lindblad, P. A. B., \& Santos-Lleó, M. 1996, A\&AS, 120, 403

Mendoza, C. 1983, in IAU Symp. 103, Planetary Nebulae, ed. D. R. Flower (Dordrecht: Reidel), 143

Moran, E. C., Barth, A. J., Kay, L. E., \& Filippenko, A. V. 2000, ApJ, 540, L73

Osterbrock, D. E. 1989, Astrophysics of Gaseous Nebulae and Active Galactic Nuclei (Mill Valley, CA: Univ. Science Books)

Rice, M. S., Martini, P., Greene, J. E., Pogge, R. W., Shields, J. C., Mulchaey, J. S., \& Regan, M. W. 2006, ApJ, 636, 654

Sarzi, M., et al. 2006, MNRAS, 366, 1151

Shu, X. W., Wang, J. X., Jiang, P., Fan, L. L., \& Wang, T. G. 2007, ApJ, 657, 167

Spergel, D. N., et al. 2003, ApJS, 148, 175

Theureau, G., et al. 1998, A\&AS, 130, 333

Ulvestad, J. S., \& Wilson, A. S. 1989, ApJ, 343, 659

Vazdekis, A. 1999, ApJ, 513, 224

Veilleux, S., \& Osterbrock, D. E. 1987, ApJS, 63, 295 